\title{
DOES SIMULTANEOUS ANTEGRADE/RETROGRADE CARDIOPLEGIA IMPROVE MYOCARDIAL PERFUSION IN THE AREAS AT RISK? A MAGNETIC RESONANCE PERFUSION IMAGING STUDY IN ISOLATED PIG HEARTS
}

Ganghong Tian, MD, $\mathrm{PhD}^{\mathrm{a}}$

Jie Shen, $\mathrm{PhD}^{\mathrm{a}}$

Jiankang Sun, MSc ${ }^{\mathrm{a}}$

Bo Xiang, DDS ${ }^{\mathrm{a}}$

Godwin I. Oriaku, BSc ${ }^{\mathrm{a}}$

Luo Zhezong, MD ${ }^{\mathrm{a}}$

Gordon Scarth, $\mathrm{PhD}^{\mathrm{a}}$

Ray Somorjai, $\mathrm{PhD}^{\mathrm{a}}$

John K. Saunders, $\mathrm{PhD}^{\mathrm{a}}$

Tomas A. Salerno, MD, MSc ${ }^{\mathrm{b}}$

Roxanne Deslauriers, $\mathrm{PhD}^{\mathrm{a}}$
Objective: This study was designed to determine whether simultaneous antegrade/retrograde cardioplegia improves myocardial perfusion in areas supplied by occluded vessels. Methods: Isolated pig hearts placed in a Langendorff preparation were divided into two groups. The left anterior descending coronary artery was occluded at its origin. In group $1(n=7)$, simultaneous antegrade/retrograde cardioplegia was conducted with use of a single perfusion unit with tubing in a Y-shaped configuration at the end, joined to the aorta and the coronary sinus. In group $2(n=8)$ simultaneous antegrade/retrograde cardioplegia was performed with two separate units, one for antegrade delivery of cardioplegic solution and the other for retrograde cardioplegic solution delivery. Myocardial perfusion in the region supplied by the left anterior descending artery and the region not supplied by this artery was assessed by magnetic resonance imaging, with use of a magnetic resonance contrast agent. The contrast agent was introduced into the common perfusion line in group 1 and into the aortic line only in group 2. Results: Magnetic resonance images showed that the myocardium in the region supported by the left anterior descending artery could not be perfused with antegrade cardioplegic solution because of occlusion of the artery. During simultaneous antegrade/retrograde cardioplegia, however, the myocardium in the left anterior descending region was perfused by approximately $40 \%$ to $50 \%$ (group 1) or $20 \%$ to $30 \%$ (group 2) of the degree of perfusion in the region not perfused by the left anterior descending artery (100\%). Almost no cardioplegic solution was delivered to the heart through the coronary sinus route during simultaneous antegrade/ retrograde cardioplegia in both groups of hearts. Myocardial perfusion in the region supported by the left anterior descending artery was heterogeneous during simultaneous antegrade/retrograde cardioplegia. Conclusions: Simultaneous antegrade/retrograde cardioplegia significantly improved myocardial perfusion in jeopardized areas of the myocardium. The jeopardized myocardium was mainly perfused by the solution drained from the adjacent normal tissue. Elevated pressure at the coronary sinus during simultaneous antegrade/ retrograde cardioplegia is responsible for the redistribution of antegradely delivered cardioplegic solution. (J Thorac Cardiovasc Surg 1998;115:913-24)
From the Institute for Biodiagnostics, National Research Council, Winnipeg, Manitoba, Canada, ${ }^{\text {a }}$ and the Division of Cardiothoracic Surgery, Faculty of Medicine, State University of New York, Buffalo, N.Y. ${ }^{\mathrm{b}}$

This work was supported in part by the Medical Research Council of Canada (grant 96090P-3408-BME-CDAA-26321). Received for publication Feb. 27, 1997; revisions requested May 7, 1997; revisions received July 9, 1997; accepted for publication Sept. 30, 1997.

Address for reprints: Ganghong Tian, MD, PhD, Institute for Biodiagnostics, National Research Council Canada, 435 Ellice Ave., Winnipeg, Manitoba, Canada R3B 1Y6.

Copyright (C) 1998 by Mosby, Inc.

$0022-5223 / 98 \$ 5.00+0 \quad \mathbf{1 2} / \mathbf{1} / \mathbf{8 6 5 1 7}$
Antegrade and retrograde cardioplegia are imporAtant techniques for protecting the heart during cardiac operations. ${ }^{1-3}$ In the presence of coronary stenosis or occlusion antegrade cardioplegia is unable to deliver a sufficient amount of solution to the areas supported by the diseased artery. ${ }^{4-6}$ Because atherosclerosis does not occur in the coronary venous system ${ }^{7,8}$ and the coronary arterial system is not necessarily the only outlet for cardioplegic solution delivered retrogradely, ${ }^{8-12}$ the jeopardized myocardium can be perfused by retrograde delivery of cardioplegic solution. Because of low flow, ${ }^{13,} 14$ het- 
erogeneous distribution, ${ }^{15}$ and poor supply to the right ventricular wall, ${ }^{16,17}$ retrograde cardioplegia has been shown to be significantly less efficient than antegrade cardioplegia for myocardial protection. ${ }^{13}$ On the basis of the advantages and disadvantages of both cardioplegic techniques, it is expected that the combination of the two techniques might become a superior cardiac protective strategy, leading to significant improvement in myocardial protection for patients at high risk. ${ }^{18,19}$ Although there are clinical and experimental studies suggesting that simultaneous antegrade/retrograde cardioplegia (SARC) provides better cardiac protection than either antegrade cardioplegia or retrograde cardioplegia, ${ }^{20,21}$ it has not been directly demonstrated that SARC improves myocardial perfusion. Relative to antegrade perfusion alone, myocardial perfusion in the healthy region is unlikely to be further improved by simultaneous antegrade/retrograde perfusion. The present study was aimed at determining whether SARC improves myocardial perfusion in jeopardized areas.

The concept of SARC might have originated from the technique of intermittent occlusion of the coronary sinus (IOCS). The latter has been used for short periods as a strategy to salvage ischemic myocardium in the beating heart and was shown to be able to reduce infarct size and improve regional myocardial function. However, it has not been directly demonstrated that IOCS indeed improves myocardial perfusion. In addition, because the conditions of the heart (beating vs arrested) and the techniques used (intermittent occlusion vs continuous perfusion of the coronary sinus) are different with these two methods, the beneficial effects observed with IOCS may not necessarily occur in SARC.

Magnetic resonance (MR) imaging shows that SARC significantly improves myocardial perfusion in the jeopardized area of the myocardium. Interestingly, almost no cardioplegic solution was delivered to the heart through the coronary sinus line during SARC under our experimental conditions. We speculate that elevated pressure at the coronary sinus during SARC redistributes the solution drained from adjacent normal tissue into the jeopardized myocardium.

\section{Material and methods}

Isolated pig heart preparation. The pig was chosen as the animal model because the venous valves and anastomoses in the pig heart are similar to those of the human heart. ${ }^{22,23}$ All animals received humane care in compliance with the "Guide to the Care and Use of Experimental Animals" (first edition) formulated by the Canadian Council on Animal Care. The protocols used in this study were approved by the National Research Council Animal Care Committee.

Domestic pigs weighing 45 to $55 \mathrm{~kg}$ were sedated with an intramuscular injection of diazepam (5 to $10 \mathrm{mg}$ ) and ketamine $(25 \mathrm{mg})$. Anesthesia was maintained with $1 \%$ to $2 \%$ isoflurane in a mixture of oxygen and nitrous oxide. The respiratory rate and volume were adjusted to keep the arterial blood gas values within the normal physiologic range. The brachiocephalic artery was cannulated at the level of the common carotid artery for arterial pressure monitoring, blood sampling, and infusion of cardioplegic solution. A sternotomy was performed. The brachiocephalic and subclavian arteries were dissected. The pericardium was opened longitudinally along the midline. The ascending aorta and the main pulmonary artery were isolated by threading umbilical tape around the origin of the descending aorta. Anticoagulation was provided by injection of heparin (3000 IU) into the superior vena cava. A cannula was inserted centrally in the brachiocephalic artery. The brachiocephalic artery, subclavian artery, descending aorta, and superior and inferior venae cavae were then clamped in succession. Heparinized cold (approximately $4^{\circ} \mathrm{C}$ ) cardioplegic solution was infused into the aortic root $(10 \mathrm{ml} / \mathrm{kg}$ body weight). The right and left atria were cut to allow drainage of the cardioplegic solution and to prevent warm blood in the lungs from returning to the heart. The heart was excised and then mounted in a Langendorff perfusion apparatus. A hydraulic occluder (IN VIVO METRIC, Healdsberg, Calif.) was placed at the origin of the left anterior descending artery (LAD). A 15F retrograde cannula (DLP Inc., Grand Rapids, Mich.) with a manually inflated balloon at the tip was positioned approximately $1 \mathrm{~cm}$ into the coronary sinus and then secured with a purse-string suture. We did not advance this cannula as far as possible because it might have impeded effluence of antegradely perfused cardioplegic solution. In this study, all of the hearts showed extension of the posterior interventricular vein. The flow of retrograde perfusion was constantly monitored with use of an in-line transonic flowmeter.

Perfusion solutions. Complete washout of the contrast agent before any subsequent injection of the agent is a prerequisite for accurate measurement of tissue perfusion. The amount of blood obtained from one pig was not sufficient to perform an imaging study. Thus in the present study we used an oxygenated crystalloid cardioplegic solution as the perfusion medium. The composition of this solution was as follows (in millimoles per liter): $\mathrm{NaCl} \mathrm{100,}$ $\mathrm{KCl} 16, \mathrm{MgCl}_{2} 16$, ethylenediaminetetraacetic acid 0.5, glucose $11, \mathrm{NaHCO}_{3} 25, \mathrm{KH}_{2} \mathrm{PO}_{4} 1.2, \mathrm{CaCl}_{2} 1.75$, and bovine serum albumin $0.5 \%$. The concentration of free calcium in this solution was 1.1 to $1.2 \mathrm{mmol} / \mathrm{L}$. The $\mathrm{pH}$ of the solution was adjusted to 7.4 to 7.5 . Tepid cardioplegic solution has been shown to improve myocardial protection during cardiac operations. We believe that this temperature may combine the advantages of both hypothermic and normothermic cardioplegia. Therefore the 


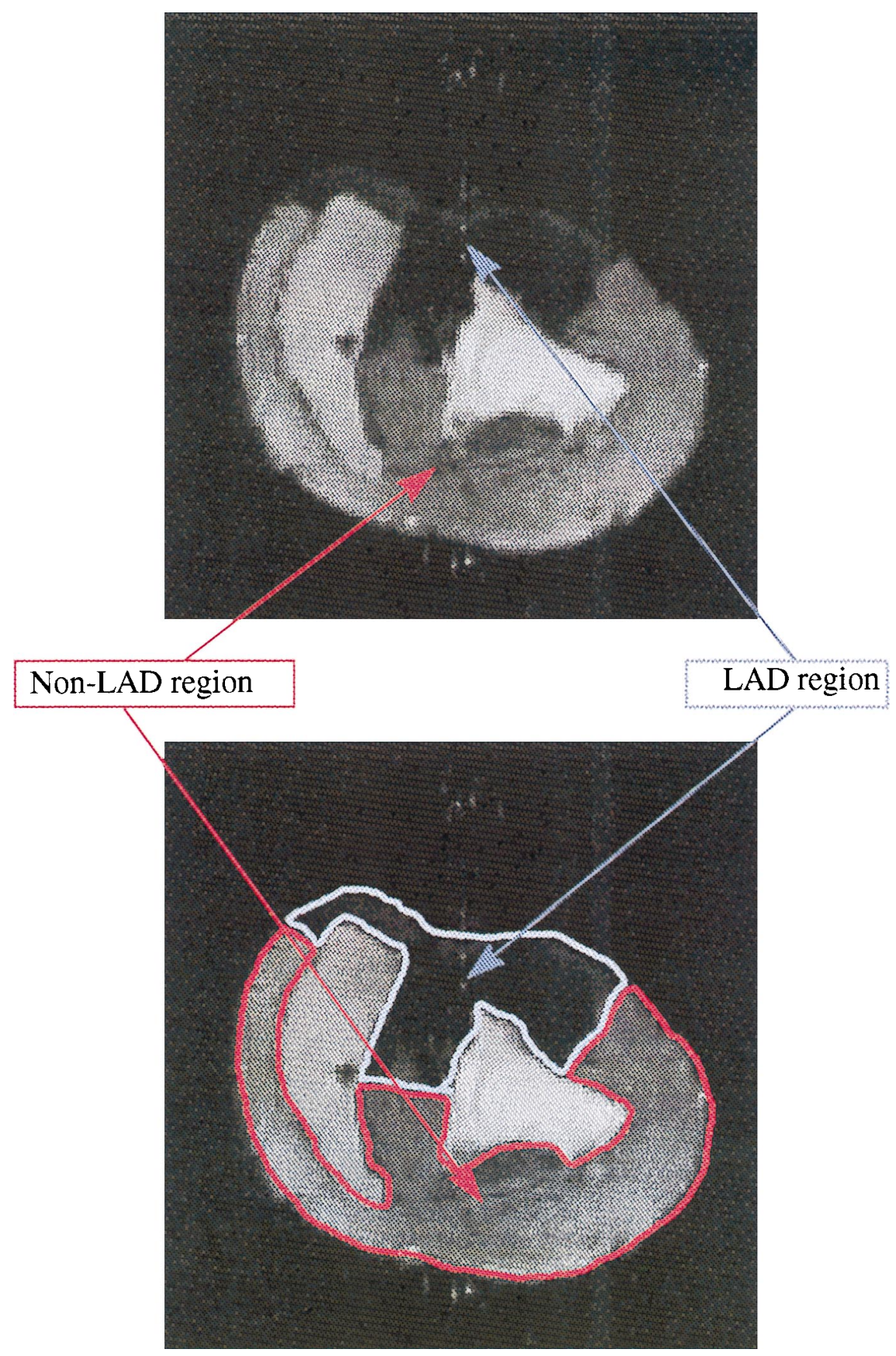

Fig. 1. Top, A representative MR perfusion image acquired along the short cardiac axis from a heart with an occluded LAD. Bottom, The same image with boundary lines defining the LAD and non-LAD regions.

temperature of the heart was maintained at $30^{\circ}$ to $31^{\circ} \mathrm{C}$ throughout the protocol.

MR perfusion imaging. Myocardial perfusion was assessed with MR imaging with an MR contrast agent (gadolinium-diethylenetriamine pentaacetic acid). This technique is noninvasive and nondestructive, such that a heart can serve as its own control. ${ }^{24,25}$ This technique also offers a very high spatial resolution and is excellent for detecting small regions of perfusion deficits.

T1-weighted MR imaging was used to assess myocardial perfusion in this study because this imaging method provides a higher magnitude of contrast agent-induced 


\section{Experimental Setups}

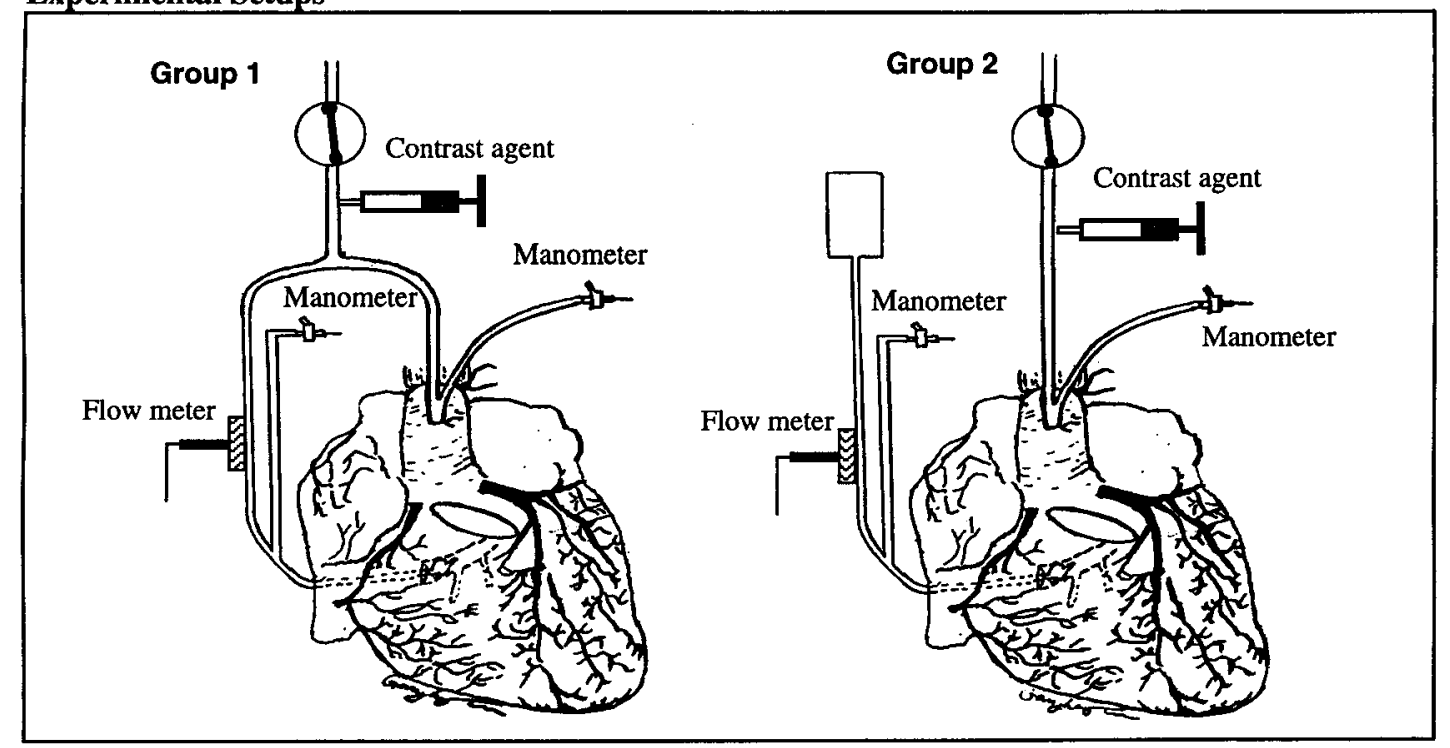

\section{Experimental Procedure}

\begin{tabular}{c|c|c|c}
$\begin{array}{l}\text { Antegrade, open LAD } \\
\text { contrast agent }\end{array}$ & $\begin{array}{l}\text { Antegrade, occluded LAD, } \\
\text { contrast agent }\end{array}$ & $\begin{array}{l}\text { SARC, occluded LAD, } \\
\text { contrast agent }\end{array}$ & $\begin{array}{l}\text { Antegrade, occluded LAD } \\
\text { no contrast agent }\end{array}$ \\
\hline MR imaging & MR imaging & MR imaging & MR imaging
\end{tabular}

Fig. 2. Experimental setups for SARC (top panel) and experimental protocol (bottom panel). Antegrade, Antegrade delivery of cardioplegic solution.

enhancement of signal intensity and has lower sensitivity to distortion caused by the interface between the myocardium and the air compared with the $\mathrm{T} 2 *$-weighted imaging method. T1-weighted imaging was performed with a 7 tesla $40 \mathrm{~cm}$ horizontal bore magnet and a Helmholtz probe surrounding the whole heart. The pulse sequence to acquire T1-weighted images was an inversion-recovery gradient-echo with a short repetition time $(10 \mathrm{msec})$ and short echo time $(4 \mathrm{msec})$. The inversion time and flip angle of the pulse were $250 \mathrm{msec}$ and $10^{\circ}$, respectively. Each image covered a $15 \mathrm{~cm}^{2}$ field of view with a $128 \times$ 128 matrix size, leading to a pixel size of $1.17 \mathrm{~mm}^{2}$. One image was acquired every 1.25 seconds. The images were acquired along the short axis of the heart. This orientation of the images provides the best view of myocardial perfusion throughout the heart with a single slice. Fifty images were taken in a block. Three blocks of images were acquired for each injection of contrast agent to follow the dynamic changes in contrast agent level and its distribution in the myocardium. Gadolinium-diethylenetriamine pentaacetic acid $(0.05 \mathrm{ml} / \mathrm{kg}$ body weight; Berlex Canada, Montreal, Canada) was used as the MR contrast agent.

Time courses of the signal intensity of the perfusion images in the LAD region and the non-LAD region were generated by averaging the signal intensities measured in each area (Fig. 1).

Experimental protocol and procedure. The experimental protocol and setups used to perform SARC for each group of hearts are illustrated in Fig. 2. The experimental protocol was divided into four periods. During the first period, the hearts were perfused antegradely with a patent LAD. In the second period, the LAD was completely occluded by inflating the occluder while the hearts were still perfused antegradely. It was expected that the MR images obtained during this period would show no perfusion in the LAD region. During the third period the LAD remained occluded and the hearts were subjected to SARC. The hypothesis was that SARC would deliver some cardioplegic solution to the LAD region but that flow to this region would be much less than that to the 


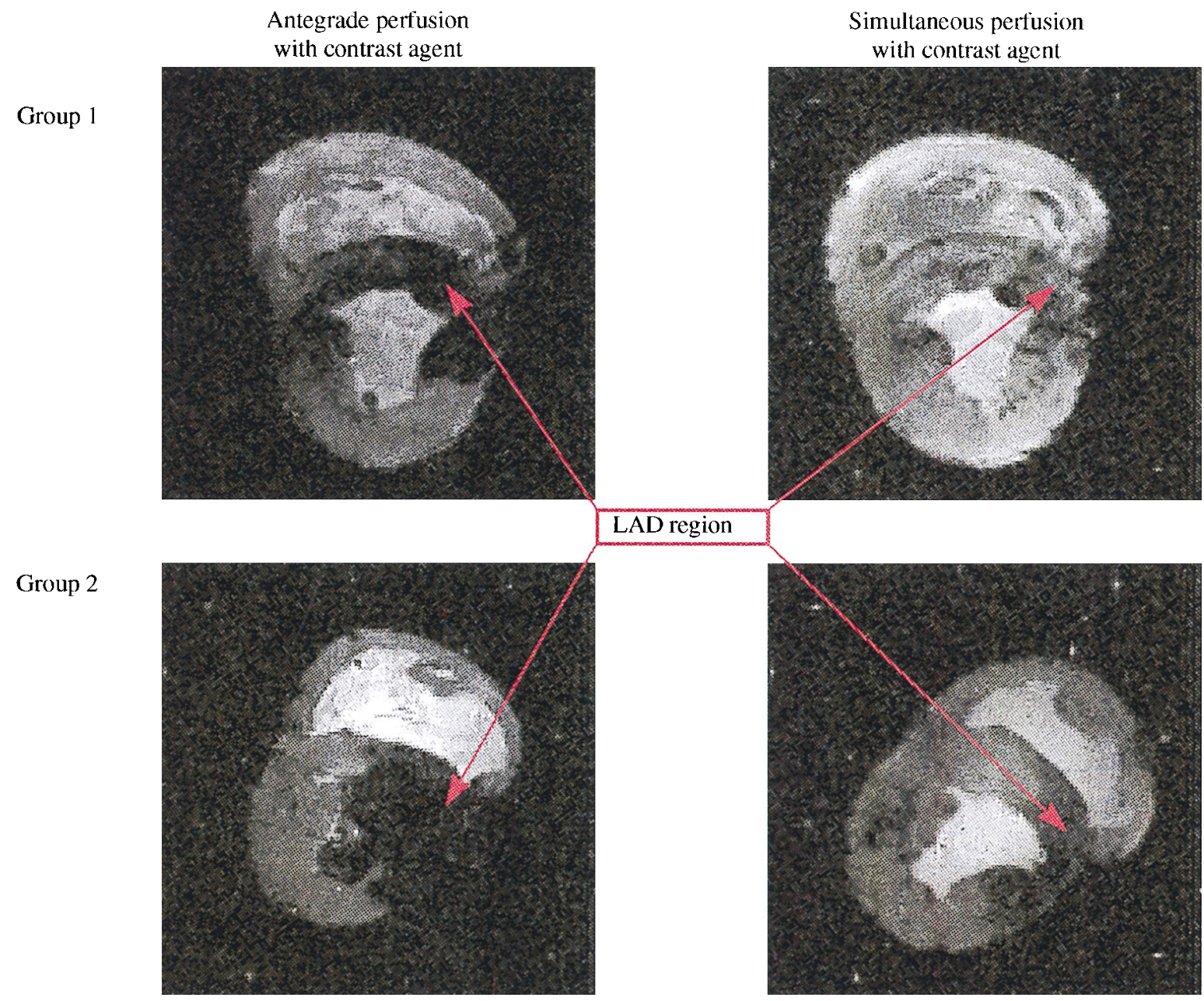

Fig. 3. Representative MR images acquired during antegrade cardioplegic solution delivery (second period) and during SARC from a group 1 heart (top images) and a group 2 heart (bottom images). No perfusion is seen in the LAD region during antegrade cardioplegic solution delivery (second period) whereas myocardium in the LAD region was partially perfused by SARC in both hearts.

non-LAD region. Accordingly, the signal intensity of the MR images was expected to be significantly lower in the $\mathrm{LAD}$ region than in the non-LAD region. To show clearly that SARC provides perfusion in the LAD region, in the fourth period antegrade perfusion was performed without contrast agent while the LAD remained occluded. The purpose was to wash out the contrast agent delivered to the non-LAD region during the previous SARC perfusion period. It was anticipated that contrast agent in the LAD region would remain in the area and MR images obtained during this period were expected to show an area of high signal intensity in the LAD region, further demonstrating that SARC can deliver cardioplegic solution to jeopardized myocardium.

In group $1(n=7)$, SARC was performed with one perfusion unit through $\mathrm{Y}$-shaped tubing with one end joined to the aorta and the other to the coronary sinus Pressure at the aorta and the coronary sinus was maintained at 40 to $50 \mathrm{~mm} \mathrm{Hg}$. In group $2(n=8)$, SARC was done with two perfusion units; one perfusion pump was used for antegrade cardioplegia, and a saline solution bag filled with the cardioplegic solution was used for retrograde cardioplegia. The pressures at the aorta and the coronary sinus were maintained at $55 \mathrm{~mm} \mathrm{Hg}$ and $38 \mathrm{~mm}$ $\mathrm{Hg}$, respectively. The MR contrast agent was injected into the common line in group 1 hearts, whereas it was administered only into the aortic line in group 2 hearts. No contrast agent was used during the fourth period in either group of hearts. Each period lasted 10 minutes.

During the interval between the first and second periods and the interval between the second and third periods, the LAD occluder was released for 5 minutes to wash out 


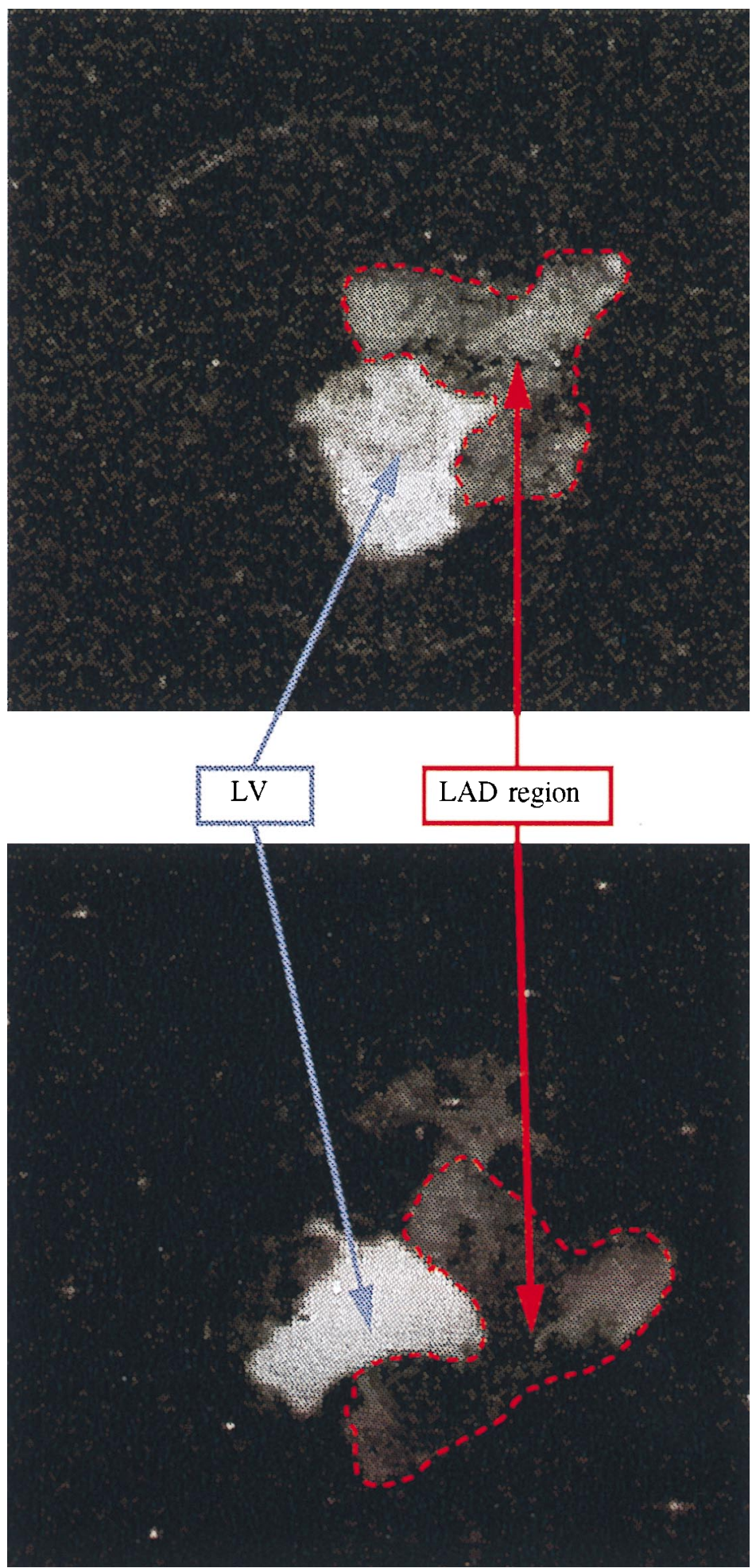

Fig. 4. Representative MR images obtained during antegrade perfusion with no contrast agent (fourth period) immediately after SARC in a group 1 heart (top image) and a group 2 heart (bottom image). The images show retention of contrast agent in the LAD region, indicating that this part of myocardium was perfused during SARC. $L V$, Left ventricle. 
the contrast agent delivered during the previous injection as well as to minimize any possible injury to the coronary vessels in the region. MR images were continuously acquired during these intervals to ensure the removal of all contrast agent from the heart before the subsequent injection.

Statistical analyses. Statistical analyses were performed with STATISTICA computer software (STATSOFT Inc., Tulsa, Okla.). All results are expressed as the mean plus or minus the standard error of the mean. Comparison of the time course of signal intensity from the LAD and non-LAD regions was performed by one-way analysis of variance with repeated measurements. Oneway analysis of variance was also used to compare the maximum signal intensity obtained from the LAD region during various stages of the protocol. The distribution of cardioplegic solution in the LAD region during SARC was determined using EvIdent two- and three-dimensional image analysis software (Institute for Biodiagnostics, NRC, Winnipeg, Canada).

\section{Results}

Fig. 3 shows representative images obtained from groups 1 (top images) and 2 (bottom images) during antegrade delivery of cardioplegic solution (second period) and SARC (third period) with an occluded LAD. As expected, the images acquired from both groups during the second period (Fig. 3, left) show no contrast agent in the LAD region, indicating that no perfusion occurred in this area during antegrade cardioplegic solution delivery with the LAD occluded. The images obtained during SARC show penetration of contrast agent into the LAD region (Fig. 3, right). This suggests that SARC delivers cardioplegic solution to the jeopardized areas of the myocardium. Furthermore, Fig. 4 shows representative images obtained during antegrade perfusion (fourth period) with the LAD occluded after SARC. No contrast agent was used during this imaging period to wash out contrast agent in the non-LAD regions administered in the previous steps. The images obtained during the fourth period show that contrast agent remained in the LAD region. This further demonstrates that the myocardium supplied by an occluded coronary artery can be perfused with the use of SARC.

In addition, in group 1 hearts the pressure values in the aorta and at the coronary sinus were similar when the coronary sinus balloon was fully inflated: $46.6 \pm 3.2 \mathrm{~mm} \mathrm{Hg}$ in the aorta and $46.7 \pm 2.9 \mathrm{~mm}$ $\mathrm{Hg}$ in the coronary sinus.

Fig. 5 summarizes myocardial perfusion during antegrade delivery of cardioplegic solution (second period, top panel) and SARC (third period, middle panel) in the LAD and the non-LAD regions in
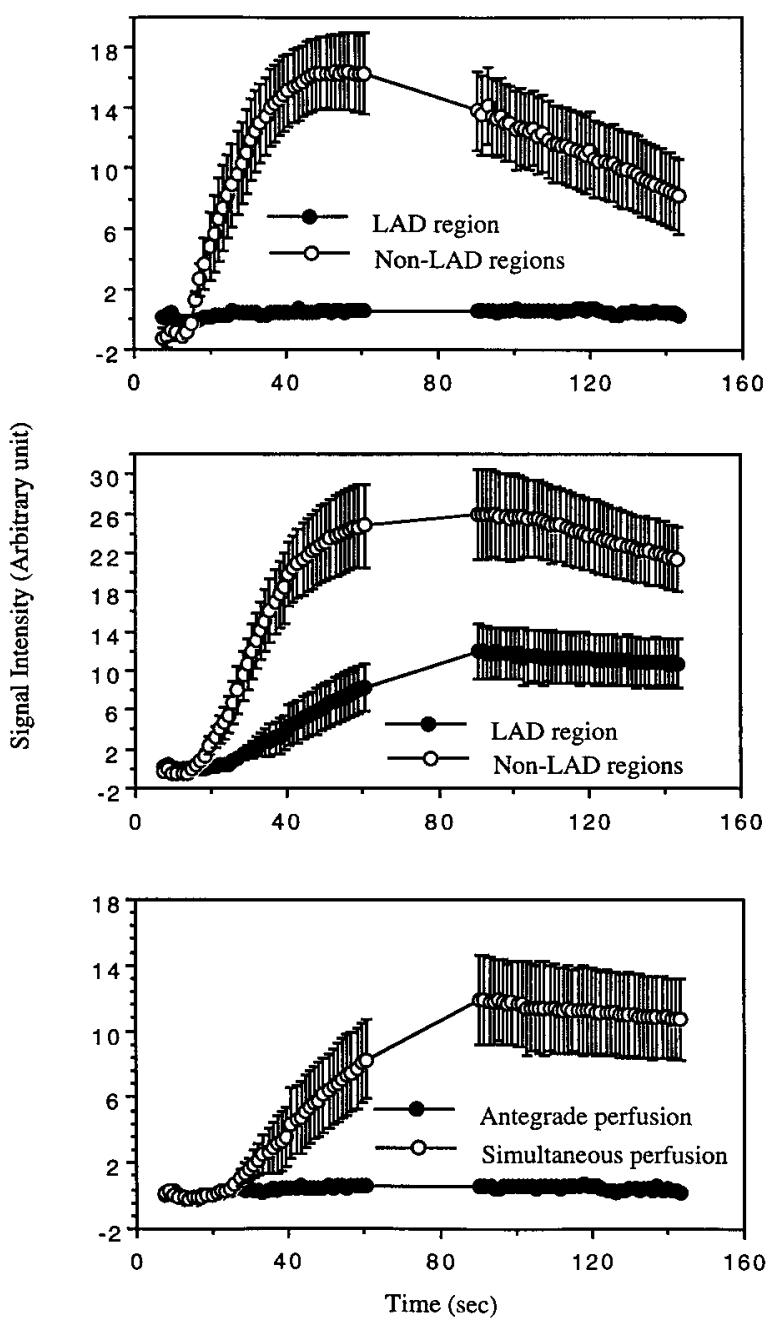

Fig. 5. Time courses of signal intensity from the LAD and non-LAD regions in group 1 hearts during antegrade cardioplegic solution delivery (second period, top panel) and SARC (middle panel). The time courses of signal intensity from the LAD region during antegrade cardioplegia and SARC are shown in the bottom panel.

group 1 hearts. During antegrade perfusion (second period), signal intensity in the LAD region remained almost unchanged whereas signal intensity in the non-LAD region showed a rapid and significant increase $(p<0.001)$, indicating that the myocardium in the LAD region was virtually not perfused. During SARC (Fig. 5, middle panel), injection of contrast agent resulted in an increase of signal intensity in both regions, although the magnitude of signal intensity increase was significantly smaller in the $\mathrm{LAD}$ region than in the non-LAD region $(p<$ $0.001)$. SARC resulted in a significant increase of 

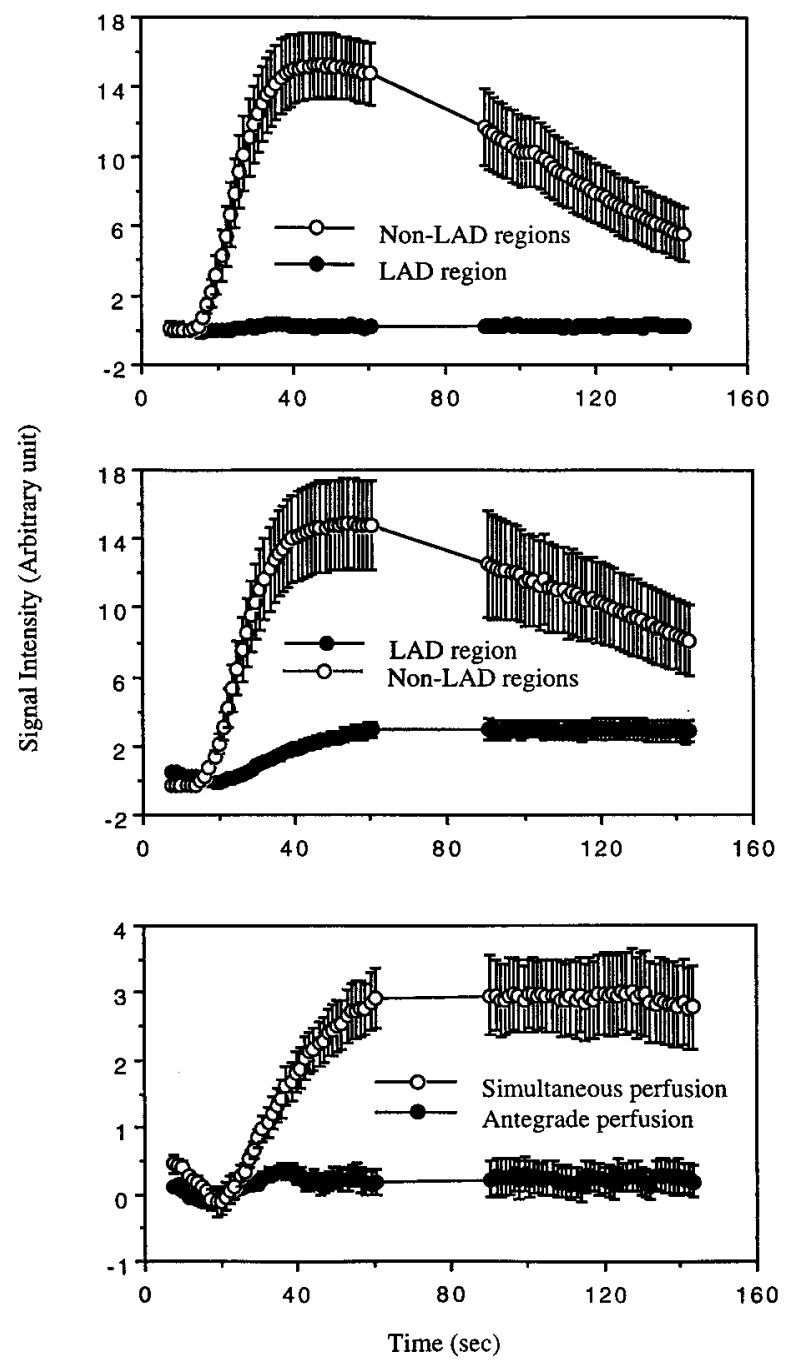

Fig. 6. Time courses of signal intensity from the LAD and non-LAD regions in group 2 hearts during antegrade cardioplegic solution delivery (second period, top panel) and SARC (middle panel). The time courses of signal intensity from the LAD region during antegrade cardioplegia and SARC are shown in the bottom panel.

signal intensity in the LAD region compared with that during antegrade cardioplegia $(p<0.001)$ (bottom panel). Although contrast agent was administered only into the aortic line in group 2 hearts, similar results were obtained and are shown in Fig. 6.

Comparisons of contrast agent-induced maximum signal intensity obtained during antegrade delivery of cardioplegic solution (second period) and SARC (third period) from the LAD region and the non-LAD regions are shown in Fig. 7. Maximum signal intensity from the non-LAD region was set as
$100 \%$. During antegrade cardioplegia, maximum signal intensity in the LAD region was $3.54 \% \pm$ $0.59 \%$ of that in the non-LAD region, which may be attributed to baseline noise. This suggests that almost no solution was delivered to the LAD region during antegrade perfusion in the presence of LAD occlusion. The maximum signal intensity in the LAD region was significantly higher $(p<0.001)$ during SARC $(34.6 \% \pm 5.7 \%$, combination of groups 1 and 2) than during antegrade cardioplegia (second period, $3.54 \% \pm 0.59 \%$ ), indicating that the LADsupported myocardium was being perfused by SARC. Furthermore, maximum signal intensity in the LAD region during SARC was significantly higher in group 1 hearts $(47.3 \% \pm 8.6 \%)$ than in group 2 hearts $(23.46 \% \pm 5.2 \%)(p=0.002)$, suggesting that pressure at the coronary sinus (relative to the pressure at the aorta) is an important factor in determining the flow of SARC solution to the jeopardized myocardium.

Interestingly, we found that no solution was delivered to the heart through the coronary sinus during SARC in group 2 hearts. In group 1, five hearts received no solution through the coronary sinus during SARC. Retrograde flow to the remaining two hearts was also extremely low (1 to 10 $\mathrm{ml} / \mathrm{min}$ ).

Representative patterns of cardioplegic distribution in the LAD region obtained during SARC are shown in Fig. 8. All the hearts in both groups showed heterogeneous perfusion to the occluded myocardium during SARC.

\section{Discussion}

SARC through various routes has been proposed as a superior technique for heart protection during cardiac operations, in particular for patients at high risk. ${ }^{18-21}$ However, it has not been directly demonstrated that SARC does indeed improve myocardial perfusion. It is also unclear whether the heart is perfused through the aorta and the coronary sinus simultaneously or only through the aorta during SARC. The present study was therefore conducted to answer these questions. We found that SARC significantly improved myocardial perfusion in the occluded myocardium and that under our experimental conditions no solution could be delivered to the heart through the retrograde line, suggesting that the improvement in myocardial perfusion with SARC was most likely a result of the increase in coronary sinus pressure.

In the hearts in group 1 SARC produced an 


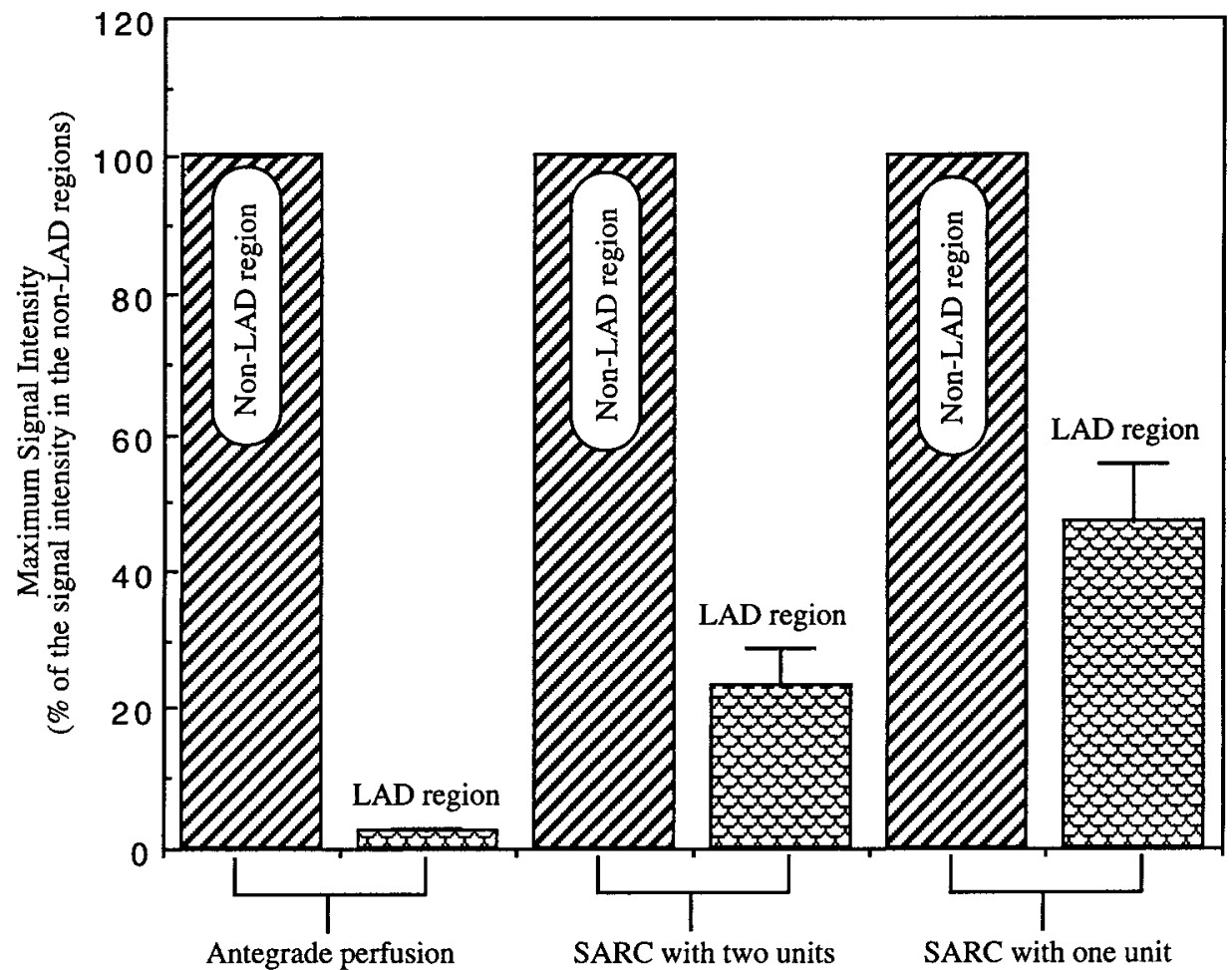

Fig. 7. Comparison of the maximum signal intensity obtained from the LAD region and the non-LAD region during the second and third periods.

increase in the maximum signal intensity in the LAD region $(47.3 \% \pm 8.6 \%)$ relative to that observed in the non-LAD region (100\%) (Fig. 7). Because it may not depend solely on the coronary flow, the maximum signal intensity cannot be used as a precise measurement of myocardial flow rate. On the other hand, comparison of the maximum signal intensity between the two regions was made in the same hearts. Other factors (which may have an impact on the maximum signal intensity, such as blood vessel volume and permeability) are expected to be comparable in these two regions. Accordingly, the ratio of the maximum signal intensity in two regions could be considered as an approximation of the ratio of myocardial flow. Based on the maximum signal intensity, myocardial flow resulting from SARC to the jeopardized myocardium is approximately $40 \%$ to $50 \%$ of that to the non-LAD region. The results demonstrate that SARC significantly improves myocardial perfusion in the jeopardized areas. On the other hand, the data also show that SARC provides heterogeneous perfusion within the occluded region (Fig. 8).

In addition, we found that pressure values in the aorta $(46.8 \pm 3.2 \mathrm{~mm} \mathrm{Hg})$ and coronary sinus $(46.7 \pm 2.9 \mathrm{~mm} \mathrm{Hg})$ were similar during SARC in group 1 hearts, if there was no solution leakage at the coronary sinus. We also found that the pressure values in the coronary sinus and retrograde flow during SARC were highly dependent on balloon inflation. If the balloon was fully inflated and the orifice of the coronary sinus was completely sealed, there was no solution leakage in the coronary sinus. Under these conditions, coronary sinus pressure reached a maximum and the retrograde flow was almost zero (five hearts showed no retrograde flow and two showed a flow rate of 1 to $10 \mathrm{ml} / \mathrm{min}$ ). This suggests that during SARC the myocardium in the LAD region was not perfused by the solution delivered through the retrograde line. It is our hypothesis that during SARC the myocardium at risk is mainly perfused by the solution delivered antegradely and drained from the adjacent tissue as a result of elevated pressure at the coronary sinus.

To test our hypothesis and to determine the effect of pressure at the coronary sinus during SARC on perfusion of the myocardium at risk, we studied the second group of hearts. In this group, contrast agent 

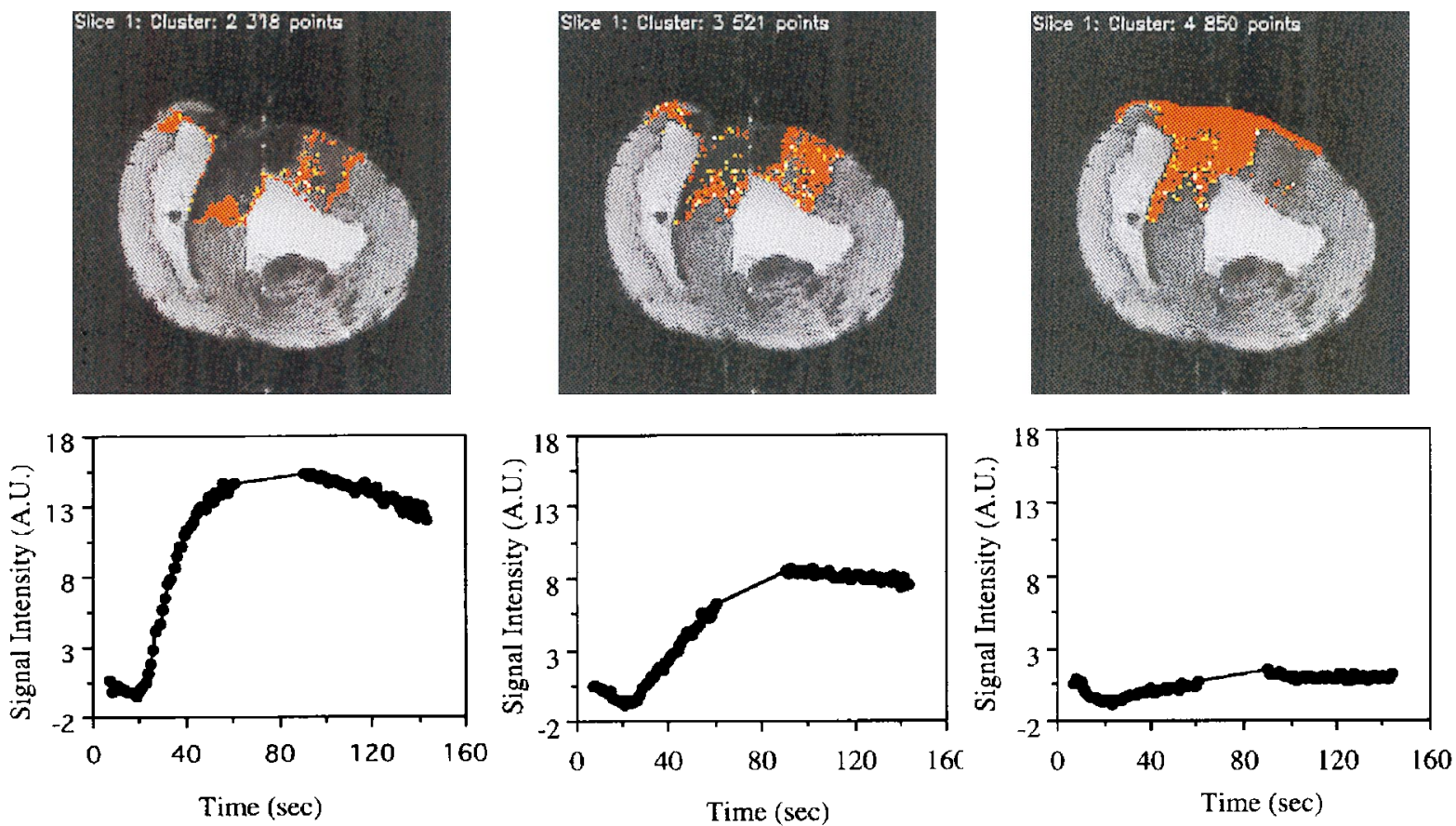

Fig. 8. A representative perfusion pattern in the LAD region during SARC. Three areas in the LAD region were identified according to their perfusion pattern (top images). The bottom graphs show the average time courses of signal intensity obtained from the corresponding areas. $A U$, Arbitrary units.

was introduced only into the aortic line during SARC. Retrograde perfusion of SARC was performed with use of a saline solution bag filled with cardioplegic solution and joined to the retrograde cannula to set the coronary sinus pressure to the designed levels. In this group, coronary sinus pressure was controlled at $38 \mathrm{~mm} \mathrm{Hg}$ and the aortic pressure was maintained at $55 \mathrm{~mm} \mathrm{Hg}$. Under these conditions, we found that no solution was delivered to the heart through the retrograde cannula during SARC. However, MR images still show invasion of MR contrast agent into the LAD region during SARC (Figs. 3, 4, 6, and 7) although no contrast agent was administered into the retrograde line. This further demonstrates that the myocardium in occluded areas is mainly perfused by the solution drained from the adjacent normal tissue, not by solution delivered retrogradely.

Furthermore, MR images show that myocardial perfusion in the LAD region was significantly less in group 2 hearts than in group 1 hearts $(23.46 \% \pm$ $5.29 \%$ vs $47.3 \% \pm 8.6 \%, p=0.002)$, suggesting that pressure at the coronary sinus relative to the aortic pressure may play an important role in determining the flow of SARC solution to the risk areas of the myocardium. We are currently testing whether a higher pressure in the coronary sinus relative to that in the aorta will further improve myocardial perfusion. In addition, maximum signal intensity in the non-LAD region from group 1 hearts was significantly higher $(p=0.024)$ during SARC than during antegrade delivery of cardioplegic solution, even though total flow and amount of contrast agent used were identical during these two periods. At present, it is still uncertain whether higher maximum signal intensity in the non-LAD region during SARC relative to that during antegrade perfusion was caused by improved perfusion or other unknown factors.

In addition, with use of our two- and three-dimensional image analysis software (EvIdent), we have demonstrated in this study that SARC does not provide homogeneous perfusion to the jeopardized myocardium. In the clinical situation, however, the size of the area at risk caused by coronary stenosis or occlusion is usually much smaller than that used in this study. Heterogeneous perfusion of SARC, therefore, may not be so pronounced in the human heart.

It must be pointed out that the present study was performed on isolated pig hearts. The subjects were healthy and relatively young. It is possible that the development of coronary anastomoses and thebesian vessels is much less in the pig heart than in the 
human patient heart. Consequently, the capacity of SARC to perfuse the area at risk may be higher in the human being than it is in the pig. In addition, this study did not compare SARC with other cardioprotective techniques (such as antegrade cardioplegia, retrograde cardioplegia, and alternate antegrade/retrograde cardioplegia) in terms of their effects on myocardial energy metabolism and functional recovery. Moreover, the potential detrimental effect of SARC on the coronary vasculature was not evaluated in this study. Therefore, at the present, no judgment can be made regarding which technique is superior for heart protection. As mentioned previously, this study was done with the use of crystalloid solution because of the insufficient blood volume that was obtainable from the animal. Because of intrinsic physiologic characteristics of blood, we expect that our study would be more clinically relevant if it had been performed with blood cardioplegic solution.

Clinically, some surgeons use antegrade delivery of cardioplegic solution through a newly established vein bypass graft while cardioplegic solution is still being delivered retrogradely through the coronary sinus. This combination has been used to test vascular anastomoses and to improve myocardial perfusion in the territory supported by the grafted artery. This is a practical and interesting combination of both antegrade and retrograde cardioplegia. However, because the conditions of this combination and those of SARC used in our study are very different, it is expected that these two techniques would result in different myocardial perfusion.

In summary, this study demonstrates that SARC improves myocardial perfusion to the areas of the myocardium at risk. However, under our experimental conditions, almost no solution is delivered to the heart through the coronary sinus. The myocardium at risk is mainly perfused by the solution drained from adjacent normal tissue during SARC. Elevated pressure in the coronary sinus seems to be the principal factor responsible for improved perfusion of the myocardium at risk.

\section{REFERENCES}

1. Donnelly AJ, Djuric M. Cardioplegia solution. Am J Hosp Pharm 1991;48:2444-60.

2. Gebhard MM, Bretschneider HJ, Schnabel PA. Cardioplegia principles and problems. In: Sperelakis N, editors. Physiology and pathophysiology of the heart. 3rd ed. Boston: Kluwer Academic; 1995. p. 731-43.

3. Takahashi A, Chambers DJ, Braimbridge MV, Hearse DJ. Car- dioplegia: relation of myocardial protection to infusion volume and duration. Eur J Cardiothorac Surg 1989:3:130-4.

4. Hilton CJ, Teubl W, Acker M, et al. Inadequate cardioplegic protection with obstructed coronary arteries. Ann Thorac Surg 1979;28:323-34.

5. Becker H, Vinten-Johansen J, Buckberg GD, Follette DM, Robertson JM. Critical importance of ensuring cardioplegic delivery with coronary stenoses. J Thorac Cardiovasc Surg 1981;81:507-15.

6. Dorsey LM, Colgan TK, Silverstein JI, Hatcher CR, Guyton RA. Alterations in regional myocardial function after heterogeneous cardioplegia. J Thorac Cardiovasc Surg 1983;86:70-9.

7. Vaage J. Retrograde cardioplegia: when and how. Scand J Thorac Cardiovasc Surg Suppl 1993;41:59-66.

8. Solorzano J, Taitelbaum G, Chiu RC. Retrograde coronary sinus perfusion for myocardial protection during cardiopulmonary bypass. Ann Thorac Surg 1978;25:201-8.

9. Salerno TA, Christakis GT, Abel J, et al. Technique and pitfalls of retrograde continuous warm blood cardioplegia. Ann Thorac Surg 1991;51:1023-5.

10. Masuda M, Yonenaga K, Katsuhisa S, Morita S, Kohno H, Tokunaga K. Myocardial protection in coronary occlusion by retrograde cardioplegic perfusion via the coronary sinus in dogs. J Thorac Cardiovasc Surg 1986;92:255-63.

11. Ratajczyk-Pakalska E. The coronary venous anatomy. In: Meerbaum S, editor. Myocardial perfusion, reperfusion, coronary venous retroperfusion. New York: Springer; 1990. p. 51-90.

12. Spaan JAE. Structure and function of coronary venous system. In: Spaan JAE, editor. Coronary blood flow. Dordrecht, The Netherlands: Kluwer Academic; 1991. p. 87-98.

13. Caldarone CA, Krukenkamp IB, Misare BD, Levitsky S. Perfusion deficits with retrograde warm blood cardioplegia. Ann Thorac Surg 1994;57:403-6.

14. Cohen MV, Matsuki T, Downey JM. Pressure-flow characteristics and nutritional capacity of coronary veins in dogs. Am J Physiol 1988;255:H834-46.

15. Tian G, Shen J, Su S, et al. Assessment of retrograde cardioplegia with magnetic resonance imaging and localized ${ }^{31} \mathrm{P}$ spectroscopy in isolated pig heart. $\mathrm{J}$ Thorac Cardiovasc Surg 1997;114:109-16.

16. Villanueva FS, Spotnitz WD, Glasheen WP, Watson DT, Jayaweera AR, Kual S. New insight into the physiology of retrograde cardioplegia delivery. Am J Physiol 1995;37: H1555-66.

17. Shiki K, Masuda M, Yonenage K, Asou T, Tokunaga K. Myocardial distribution of retrograde flow through the coronary sinus of the excised normal canine heart. Ann Thorac Surg 1986;41:265-71.

18. Ihnken K, Morita K, Buckberg GD, et al. The safety of simultaneous arterial and coronary sinus perfusion: experimental background and initial clinical results. J Card Surg 1994;9:15-25.

19. Partington MT, Acar C, Buckberg GD, Julia PL. Studies of retrograde cardioplegia: II-advantages of antegrade/retrograde cardioplegia to optimize distribution in jeopardized myocardium. J Thorac Cardiovasc Surg 1989;97:61322.

20. Shirai T, Rao V, Weisel RD, et al. Antegrade and retrograde cardioplegia: alternate or simultaneous? J Thorac Cardiovasc Surg 1996;112:787-96.

21. Bhayana JN, Kalmbach T, Booth FVM, et al. Combined 
antegrade/retrograde cardioplegia for myocardial protection: a clinical trial. J Thorac Cardiovasc Surg 1989;98:956-60.

22. Geary GG, Smith GT, McNamara JJ. Defining the anatomic perfusion bed of an occluded coronary artery and the region at risk to infarction: a comparative study in the baboon, pig and dog. Am J Cardiol 1981;47:1240-7.

23. Kong Y, Chen JTT, Zeff HJ, Whalen RE, McIntosh HE. Natural history of experimental coronary occlusion in pig: a serial cineangiographic study. Am Heart J 1969;77:4554.

24. Saeed M, Wendland MF, Higgins CB. The developing role of magnetic resonance contrast media in the detection of ischemic heart disease. Proc Soc Exp Biol Med 1995;208:238-54.

25. Wilke N, Kroll K, Merkle H, et al. Regional myocardial blood volume and flow: first-pass MR imaging with polylysine-Gd-DTPA. J Magn Reson Med 1995;5:227-37. 